\title{
Targeted chemotherapy for subcutaneous and orthotopic non- small cell lung tumors with cyclic RGD-functionalized and disulfide-crosslinked polymersomal doxorubicin
}

\author{
Yan Zou ${ }^{1,2}$, Jingjing $\mathrm{Wei}^{1}$, Yifeng $\mathrm{Xia}^{1}$, Fenghua Meng ${ }^{1}$, Jiandong Yuan ${ }^{3}$ and Zhiyuan Zhong ${ }^{1}$
}

\begin{abstract}
Lung cancer, with its high mortality and increasing morbidity, has become one of the most lethal malignancies worldwide. Here, we developed cyclic RGD peptide-directed and disulfide-crosslinked polymersomal doxorubicin (cRGD-PS-Dox) as a targeted chemotherapy for human non-small cell lung cancer (NSCLC). Notably, cRGD-PS-Dox exhibited a high Dox loading (15.2 wt.\%), small hydrodynamic diameter $(96 \mathrm{~nm})$, superb stability, prominent targetability to $a_{v} \beta_{3}$ integrin overexpressing A549 human lung cancer cells, and rapid release of the drug into nuclei, leading to a significantly improved antitumor activity compared with the control groups, i.e., PS-Dox and Lipo-Dox (a liposome injection employed in clinical settings). The pharmacokinetic and biodistribution results for CRGD-PS-Dox revealed similar elimination half-lives but two-fold enhanced tumor accumulation compared with PS-Dox and Lipo-Dox. Intriguingly, cRGD-PS-Dox effectively suppressed the growth of A549 lung tumors in both subcutaneous and orthotopic models with minimal adverse effects at a Dox dose of $12 \mathrm{mg} / \mathrm{kg}$, leading to significant survival benefits compared with PS-Dox and Lipo-Dox. This $a_{v} \beta_{3}$ integrin-targeting multifunctional polymersomal doxorubicin is highly promising for targeted chemotherapy of human NSCLC.
\end{abstract}

Signal Transduction and Targeted Therapy (2018)3:32; https://doi.org/10.1038/s41392-018-0032-7

\section{INTRODUCTION}

Lung cancers have high mortality and increasing morbidity and have become one of the most lethal malignancies worldwide. ${ }^{1-3}$ Despite the severe side-effects, chemotherapy with potent drugs such as erlotinib, gemcitabine, cisplatin, irinotecan, doxorubicin hydrochloride (Dox. $\mathrm{HCl}$ ), and pemetrexed continues to serve as the primary treatment and supportive care for lung cancer patients. ${ }^{4-6}$ During the past decade, diverse anticancer nanomedicines have been investigated to increase anticancer efficacy while decreasing the adverse effects of chemotherapeutic drugs. ${ }^{7-18}$ In a phase II clinical trial, Genexol-PM, a micellar paclitaxel formulation, in combination with gemcitabine, demonstrated favorable antitumor activity in non-small cell lung cancer (NSCLC) patients. ${ }^{19}$ To augment the tumor targetability, nano-drugs have been decorated with lung cancer cell-specific ligands, such as anisamide and peptides (e.g., cNGQGEQ and CSNIDARAC). ${ }^{20-23}$ Nonetheless, these "targeted" systems show only a moderate improvement in therapeutic efficacy, partially due to low in vivo stability and/or slow release of drugs intracellularly. Interestingly, disulfide-crosslinked nanomedicines have demonstrated excellent in vivo stability and fast intracellular drug release. ${ }^{24-28}$ We have described that disulfide-crosslinked chimeric polymersomes containing poly(ethylene glycol) on the outer surface and polyethylenimine mainly in the lumen efficiently mediated targeted delivery of methotrexate sodium (MTX.2Na) to lung tumor xenografts in nude mice. ${ }^{29}$
Here, we report on CRGD peptide-directed and disulfidecrosslinked polymersomal doxorubicin (cRGD-PS-Dox) for active targeting chemotherapy of human lung cancer xenografts in mice (Fig. 1a). PS-Dox has a vesicular structure, small size, and high loading of Dox. $\mathrm{HCl}^{21}$ similar to pegylated liposomal doxorubicin (Doxil or Caelyx) used in the clinic. ${ }^{30-35}$ CRGD was selected as a targeting ligand because both angiogenic endothelial cells and A549 human lung cancer cells overexpress $\alpha_{v} \beta_{3}$ integrins. ${ }^{36-40}$ Our results show that CRGD-PS-Dox effectively suppresses the growth of A549 lung tumors in both subcutaneous and orthotopic models in mice, resulting in clear survival benefits over PS-Dox and LipoDox.

\section{RESULTS}

Construction of cRGD-PS-Dox

Small CRGD-PS $(\sim 82 \mathrm{~nm})$ with a low polydispersity index (PDI = $0.10)$ was obtained by co-self-assembly of poly(ethylene glycol)- $b$ poly(trimethylene carbonate-co-dithiolane trimethylene carbonate) (PEG-P(TMC-DTC), $\left.\quad M_{\mathrm{n}}=5.0-24.2 \mathrm{~kg} / \mathrm{mol}\right)$ and CRGDfunctionalized PEG-P(TMC-DTC) (CRGD-PEG-P(TMC-DTC), $M_{\mathrm{n}}=$ $6.5-24.5 \mathrm{~kg} / \mathrm{mol}$ ) block copolymers $(4 / 1, \mathrm{~mol} / \mathrm{mol})$ in phosphate buffer (PB, pH 7.4, $10 \mathrm{mM}$ ) (Fig. 1b). Transmission electron microscope (TEM) micrograph corroborated a vesicular morphology and small size for CRGD-PS (Fig. 1b, inset). UV spectra showed a significant decrease in the characteristic absorbance of

\footnotetext{
${ }^{1}$ Biomedical Polymers Laboratory, College of Chemistry, Chemical Engineering and Materials Science, Soochow University, Suzhou 215123, P. R. China; ${ }^{2}$ International Joint Centre

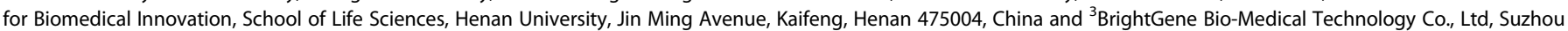
215123, P. R. China

Correspondence: Fenghua Meng (fhmeng@suda.edu.cn) or Zhiyuan Zhong (zyzhong@suda.edu.cn)
}

Received: 17 July 2018 Revised: 21 September 2018 Accepted: 7 November 2018

Published online: 14 December 2018 
a

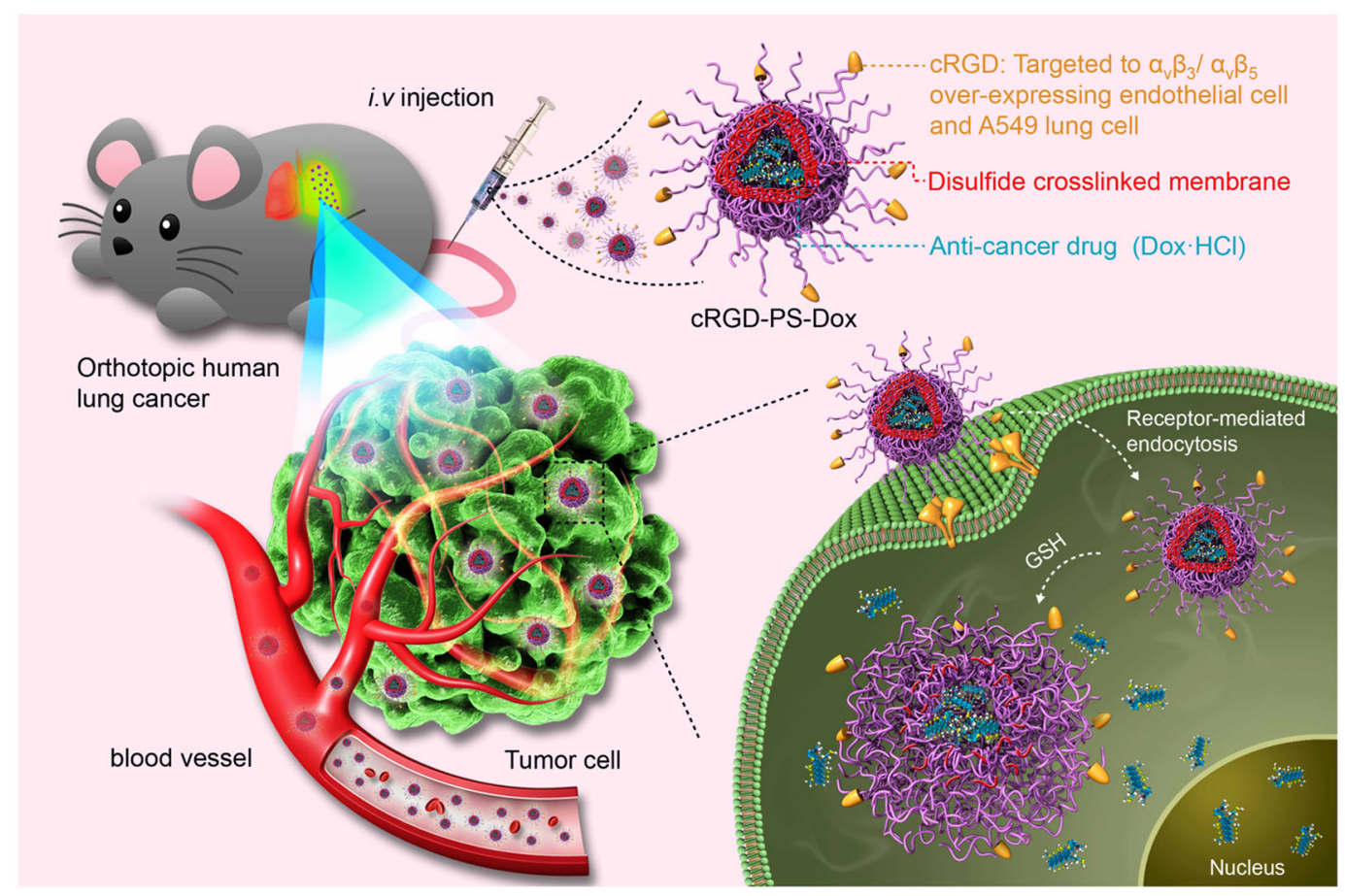

b

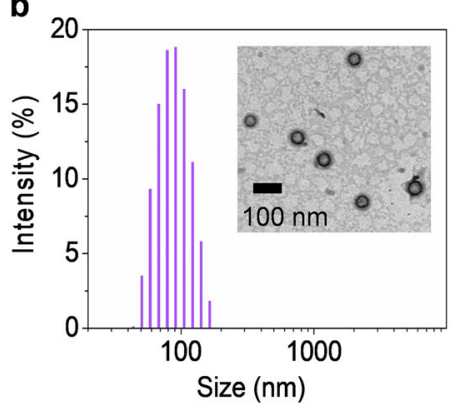

C

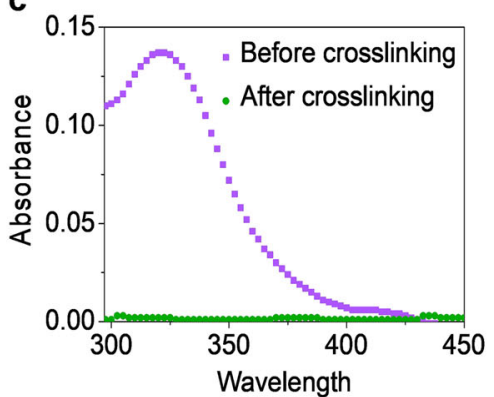

d

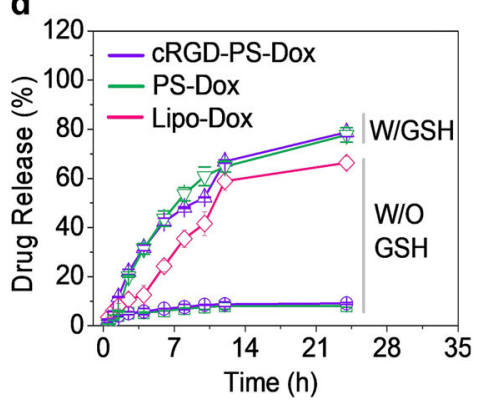

Fig. 1 a Illustration of CRGD-directed polymersomal doxorubicin (cRGD-PS-Dox) for active targeting chemotherapy of lung tumor xenografts in mice; b DLS measurement and TEM photograph of CRGD-PS; c UV absorbance of CRGD-PS before and after crosslinking; $\mathbf{d}$ In vitro Dox release in PB with or without GSH $(10 \mathrm{mM})$ at $37^{\circ} \mathrm{C}$

dithiolanes at $330 \mathrm{~nm}$ in CRGD-PS (Fig. 1c), signifying that dithiolanes underwent spontaneous ring-opening polymerization forming linear polydisulfides that crosslinked the vesicular membrane. Accordingly, cRGD-PS-Dox and PS-Dox did not have a critical vesicle concentration (CVC).

As previously reported, Dox $\cdot \mathrm{HCl}$ could be laden into $\mathrm{PS}$ by a $\mathrm{pH}-$ gradient method with high efficiency, ${ }^{41}$ and a drug loading content (DLC) of $15.2 \mathrm{wt}$ \% was achieved for CRGD-PS-Dox, which exhibited a hydrodynamic diameter of $96 \mathrm{~nm}(P D I=0.13$, Table 1). Notably, less than $15 \%$ of drug was released from cRGD-PS-Dox in $24 \mathrm{~h}$ at $\mathrm{pH} 7.4$ and $37^{\circ} \mathrm{C}$ under $2 \mu \mathrm{M} \mathrm{GSH}$ (Figure S1), indicating a high stability of CRGD-PS-Dox in circulation. The Dox release from CRGD-PS-Dox increased to approximately $46 \%, 62 \%$, and $80 \%$ upon increasing GSH concentrations to 2,5 and $10 \mathrm{mM}$, respectively (Fig. 1d \& Figure S1). In comparison, $67 \%$ Dox was released from Lipo-Dox in $24 \mathrm{~h}$ under physiological conditions. These results suggest that CRGD-PS-Dox might be robust in the circulation while quickly releasing drug in the cytoplasm.

In vitro assessment of CRGD-PS-Dox

MTT assays demonstrated that blank polymersomes, either targeted or non-targeted, were non-toxic to $a_{v} \beta_{3}$ integrinoverexpressing human lung cancer A549 cells at tested concentrations ( 1 and $2 \mathrm{mg} / \mathrm{mL}$, Fig. $2 \mathrm{a}$ ), signifying their low cytotoxicity.

\begin{tabular}{|c|c|c|c|c|c|}
\hline \multirow[t]{2}{*}{ Polymersomes } & \multicolumn{2}{|c|}{$\mathrm{DLC}^{\mathrm{a}}(\mathrm{wt} . \%)$} & \multirow[b]{2}{*}{$\operatorname{DLE}^{\mathrm{a}}(\%)$} & \multirow[b]{2}{*}{$\operatorname{Size}^{b}(n m)$} & \multirow[b]{2}{*}{$\mathrm{PDI}^{\mathrm{k}}$} \\
\hline & Theory & Determined & & & \\
\hline \multirow[t]{3}{*}{ PS-Dox } & 9.1 & 6.7 & 72.1 & 85 & 0.08 \\
\hline & 16.7 & 12.0 & 68.4 & 88 & 0.11 \\
\hline & 23.1 & 15.5 & 61.3 & 94 & 0.13 \\
\hline \multirow[t]{3}{*}{ cRGD-PS-Dox } & 9.1 & 6.8 & 73.5 & 82 & 0.06 \\
\hline & 16.7 & 11.8 & 67.2 & 86 & 0.12 \\
\hline & 23.1 & 15.2 & 59.6 & 96 & 0.13 \\
\hline
\end{tabular}

In contrast, CRGD-PS-Dox exhibited potent inhibitory effects on A549 cells with an $\mathrm{IC}_{50}$ of $3.2 \mu \mathrm{g} / \mathrm{mL}$, which was 4.1 - and 6.4 -fold lower than PS-Dox and Lipo-Dox, respectively (Fig. 2b). Notably, a comparable cytotoxicity profile was discerned for CRGD-PS-Dox and PS-Dox in $a_{v} \beta_{3}$ integrin low-expressing human MCF-7 breast cancer cells (Figure S2a). Flow cytometric analyses confirmed that 
a

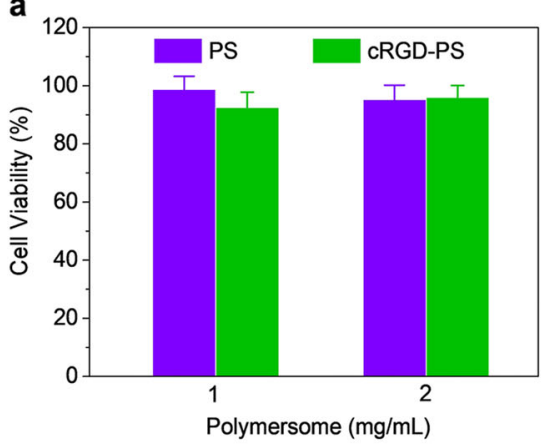

b

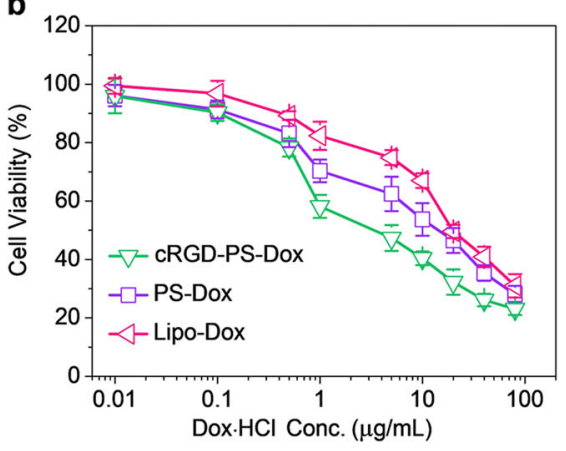

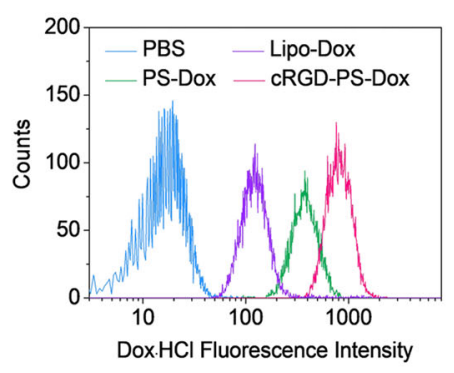

d

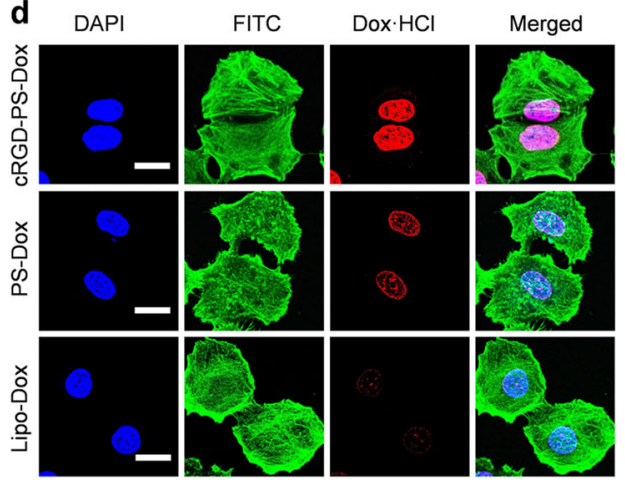

Fig. 2 a Cytotoxicity of blank cRGD-PS to A549 cells following $48 \mathrm{~h}$ incubation. b Viability of A549 cells after a $4 \mathrm{~h}$ incubation with cRGD-PSDox plus $44 \mathrm{~h}$ culture in fresh media. c Flow cytometry of $\mathrm{A} 549$ cells after a $4 \mathrm{~h}$ incubation with different formulations $(10.0 \mu \mathrm{g} / \mathrm{mL} \mathrm{Dox} \cdot \mathrm{HCl})$. d CLSM of A549 cells treated with different formulations for $4 \mathrm{~h}(10.0 \mu \mathrm{g} / \mathrm{mL} \mathrm{Dox} \cdot \mathrm{HCl})$ and further cultured in fresh media for $4 \mathrm{~h}$. The cell nuclei and cytoskeleton were stained by DAPI and phalloidin-FITC, respectively. Scale bars: $25 \mu \mathrm{m}$

the intracellular Dox.HCl intensity in CRGD-PS-Dox treated A549 cells was stronger compared with those of PS-Dox or Lipo-Dox (Fig. 2c). In MCF-7 cells, however, cRGD-PS-Dox exhibited similar cellular uptake to PS-Dox (Figure S2b), verifying that cRGD-PS-Dox has a high specificity to $a_{v} \beta_{3}$ integrin-overexpressing cancer cells. CLSM observation displayed intensive Dox. $\mathrm{HCl}$ fluorescence in the nuclei of A549 cells treated with CRGD-PS-Dox (Fig. 2d). In contrast, PS-Dox showed weak fluorescence in the nuclei and Lipo-Dox showed negligible fluorescence. The enhanced nucleic Dox release observed for CRGD-PS-Dox is likely due to its efficient uptake by $A 549$ cells via a receptor-mediated mechanism and triggered drug release in the cytoplasm.

In vivo pharmacokinetics and biodistribution of CRGD-PS-Dox In vivo stability is vital for tumor accumulation and the anti-tumor efficacy of nanotherapeutics. ${ }^{42,43}$ The Dox. $\mathrm{HCl}$ concentration in plasma was monitored over time after a single injection of CRGDPS-Dox or PS-Dox into healthy mice. The results showed that they had an elimination half-lives $\left(t_{1 / 2, \beta}\right)$ of approximately $7.5 \mathrm{~h}$ and a high area under curve (AUC) comparable to that of Lipo-Dox (Fig. 3a). To visualize their tumor accumulation, Cy7-labeled CRGDPS or PS was injected into nude mice inoculated with A549 tumor xenografts subcutaneously and observed over time using an IVIS system. Figure $3 \mathrm{~b}$ showed that tumor accumulation of cRGD-PS became obvious at $1 \mathrm{~h}$ post-injection, reached a maximum at $12 \mathrm{~h}$ and remained high at $48 \mathrm{~h}$. Notably, the liver uptake reduced to a low level after $24 \mathrm{~h}$. In comparison, Cy7-labeled PS accumulated significantly less at tumor sites despite the similar pharmacokinetics of CRGD-PS and PS, indicating the decisive role of active targeting in tumor accumulation, cell uptake and retention.

Taking advantage of the fluorescent nature of free Dox, we then investigated the biodistribution of CRGD-PS-Dox by ex vivo fluorescence imaging. The tumor and major organs were excised at $12 \mathrm{~h}$ post-injection and imaged using a fluorescence imaging system. Figure $3 c$ shows that for CRGD-PS-Dox treated mice, tumors had more intense Dox. HCl fluorescence than healthy organs, supporting selective uptake by A549 tumors, rapid cellular internalization and rapid Dox release. In sharp contrast, PS-Dox and Lipo-Dox groups showed comparably weaker Dox $\mathrm{HCl}$ fluorescence in tumors. The quantitative measurements of Dox. $\mathrm{HCl}$ demonstrated that tumor accumulation of CRGD-PS-Dox could reach approximately $7.37 \%$ of the injected dose per gram of tissue $(\% \mathrm{ID} / \mathrm{g})$, which was approximately 1.9 -fold better than the Lipo-Dox group and 2.4-fold better than the PS-Dox group (Fig. $3 d$ ). The summary of tumor-to-normal tissue $(\mathrm{T} / \mathrm{N})$ ratios revealed that $C R G D-P S-D o x$ treatment significantly reduced the Dox. $\mathrm{HCl}$ amount in major organs compared to Lipo-Dox and PSDox (Table S1). The above results suggest that CRGD functionalization of PS-Dox remarkably enhances tumor accumulation and retention. ${ }^{4,45}$ The tolerability studies revealed that cRGD-PS-Dox did not cause significant body weight loss at a Dox.HCl dose of 100 or $150 \mathrm{mg} / \mathrm{kg}$ while Lipo-Dox induced high toxicity at $20 \mathrm{mg} /$ $\mathrm{kg}$ (Figure S3), indicating that CRGD-PS-Dox has at least a 7.5-fold higher maximum-tolerated dose (MTD) than Lipo-Dox.

In vivo therapeutic administration of nude mice bearing subcutaneous A549 tumor xenografts

In vivo antitumor activity of CRGD-PS-Dox was assessed in mice bearing subcutaneous $A 549$ xenografts at a Dox. $\mathrm{HCl}$ equivalent dose of 6 or $12 \mathrm{mg} / \mathrm{kg}$ administered on day $0,4,8$, and 12 . The results demonstrated a dose-dependent tumor growth inhibition by CRGD-PS-Dox. Tumor progression was greatly suppressed at 12 $\mathrm{mg} / \mathrm{kg}$ (Fig. 4a). At $6 \mathrm{mg} / \mathrm{kg}$, cRGD-PS-Dox exhibited similar efficacy to Lipo-Dox while significantly better tumor suppression than PS-Dox, suggesting that CRGD-PS-Dox can actively target A549 tumors in vivo. Figure $4 \mathrm{~b}$ reveals that Lipo-Dox caused severe systemic toxicity with substantial loss in body weight (up to $18 \%$ ) during treatment. In contrast, for CRGD-PS-Dox and PS-Dox 
a

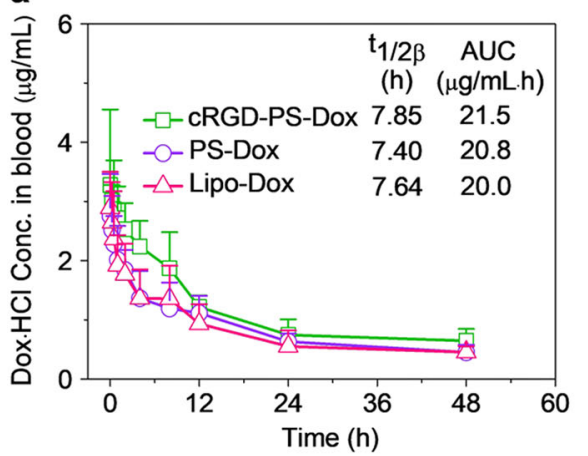

C
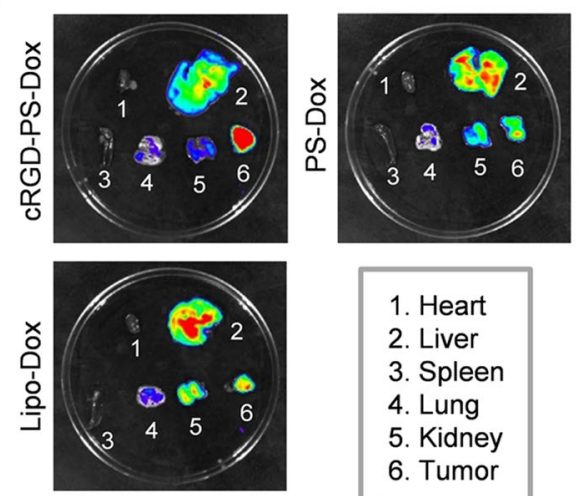

b

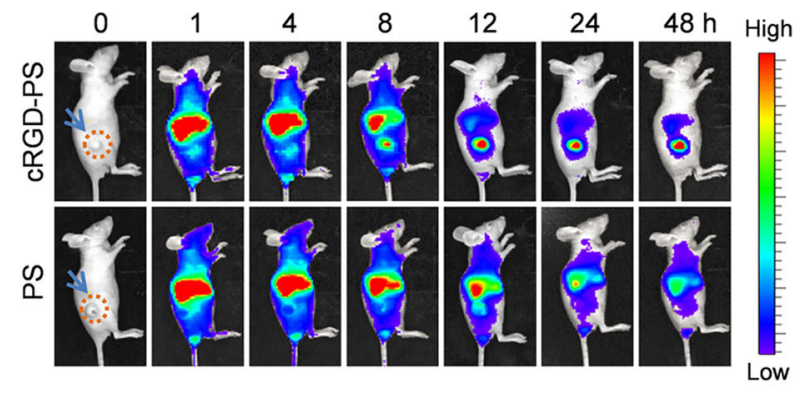

d
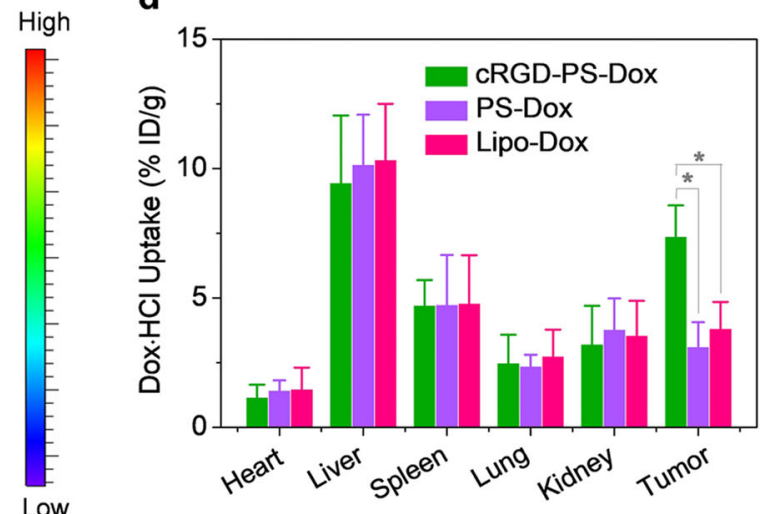

Fig. 3 a In vivo pharmacokinetics of CRGD-PS-Dox, PS-Dox and Lipo-Dox in tumor-free mice. $\mathbf{b}$ In vivo fluorescence images of mice bearing subcutaneous A549 tumors after iv injection of Cy7-labeled CRGD-PS or PS. c Ex vivo fluorescence images of tumors and major organs isolated at $12 \mathrm{~h}$ post-injection. d Quantitative drug biodistribution in tumors and major organs. ${ }^{*} p<0.05$ based on one-way ANOVA and Tukey multiple comparisons tests. For $\mathbf{a}$ and $\mathbf{d}$, the Dox. $\mathrm{HCl}$ concentration was measured by fluorescence spectroscopy and are presented as the mean $\pm \mathrm{SD}$ $(n=3)$

treated mice, their body weights had little alteration, indicating that polymersomal Dox has negligible adverse effects. The pictures of tumors collected on day 20 confirmed that mice treated with CRGD-PS-Dox at $12 \mathrm{mg} / \mathrm{kg}$ bore the smallest tumors (Fig. 4c). Figure 4d demonstrates that CRGD-PS-Dox had a remarkable tumor inhibition rate (TIR) of $82.4 \%$ at $12 \mathrm{mg} / \mathrm{kg}$. Moreover, at $6 \mathrm{mg} / \mathrm{kg}$ cRGD-PS-Dox exhibited a TIR similar to LipoDox though significantly higher than PS-Dox. These results clearly show that CRGD-PS-Dox has a markedly improved safety profile, tumor targetability and therapeutic efficacy over Lipo-Dox in A549 tumor-bearing nude mice.

In vivo treatment of nude mice bearing orthotopic A549-Luc tumor xenografts

The exceptional therapeutic benefits of cRGD-PS-Dox in the treatment of subcutaneous lung tumor models encouraged us to further assess its targetability and antitumor effects in orthotopic human A549 lung tumor xenografts in mice, which was established by implanting bioluminescent A549-Luc cancer cells into the lungs of nude mice $(n=6)$. The growth of tumors was monitored using the IVIS imaging system. All of the mice had similar luminescence intensity initially, and CRGD-PS-Dox $(12 \mathrm{mg} /$ $\mathrm{kg}$ ) induced the best tumor inhibition over a treatment period of 16 days. At $6 \mathrm{mg} / \mathrm{kg}$, CRGD-PS-Dox resulted in better tumor suppression than PS-Dox, as observed in the subcutaneous model (Fig. 5a). The quantification of lung bioluminescence intensity showed that CRGD-PS-Dox $(12 \mathrm{mg} / \mathrm{kg})$ completely suppressed tumor growth. CRGD-PS-Dox led to similar tumor inhibition as Lipo-Dox while CRGD-PS-Dox performed significantly better than PS-Dox (Fig. 5b). Notably, the body weights only slightly changed for CRGD-PS-Dox or PS-Dox treated mice while Lipo-Dox and PBS groups displayed significant body weight loss over 16 days (Fig. 5c), confirming that CRGD-PS-Dox has few side effects and can effectively retard tumor invasion into the lung. Consistently, CRGD-PS-Dox $(12 \mathrm{mg} / \mathrm{kg})$ treated mice survived the longest with a median survival time of 57 days (Fig. $5 \mathrm{~d}$ ). Meanwhile, at $6 \mathrm{mg} / \mathrm{kg}$, CRGD-PS-Dox also exhibited a clear survival benefit over Lipo-Dox and PS-Dox. On day 16, the major organs were collected from one mouse in each group for ex vivo imaging, in which the lowest bioluminescence was observed in the lung of the mouse treated with $12 \mathrm{mg} / \mathrm{kg}$ CRGD-PS-Dox (Fig. 6a). Of note, no metastasis to the liver was observed with $12 \mathrm{mg} / \mathrm{kg}$ CRGD-PS-Dox, which is in great contrast to the mice treated with the other formulations. CRGD-PS-Dox at $12 \mathrm{mg} / \mathrm{kg}$ can effectively target and suppress the growth and metastasis of orthotopic A549 lung tumors with few adverse effects, outperforming PS-Dox and Lipo-Dox.

\section{DISCUSSION}

Lung cancer is a rapidly growing human malignancy. CRGD induces significantly enhanced imaging of lung tumors via binding to $a_{v} \beta_{3}$ integrins. ${ }^{46-48}$ In this study, we investigated the targetability and anti-tumor activity of CRGD-PS-Dox in mice with subcutaneous or orthotropic A549 tumors. The pharmacokinetics studies demonstrated that CRGD-PS-Dox can circulate in the blood, comparable to Lipo-Dox. The biodistribution of CRGD-PSDox reveals a much higher drug accumulation in tumors $(7.37 \%$ $\mathrm{ID} / \mathrm{g}$ ) than that of PS-Dox or Lipo-Dox, validating that CRGD functionalization enables active targeting to A549 tumors in vivo. ${ }^{40,49}$ Interestingly, cRGD-PS-Dox exhibits an extraordinarily high MTD (over $150 \mathrm{mg} / \mathrm{kg}$ ) that is over 7.5 -fold higher than that of Lipo-Dox. Therefore, CRGD-PS-Dox has a much broader 


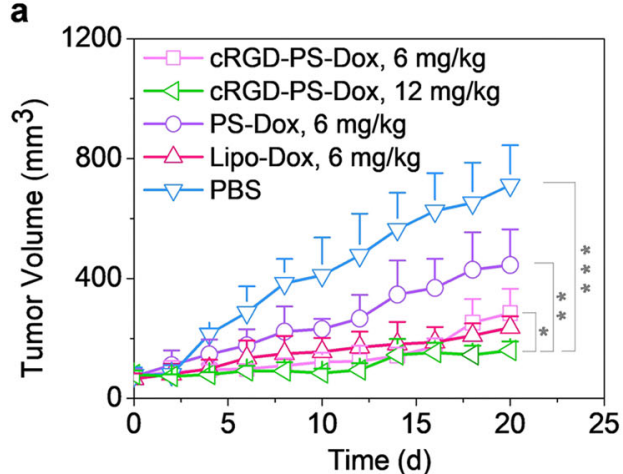

C

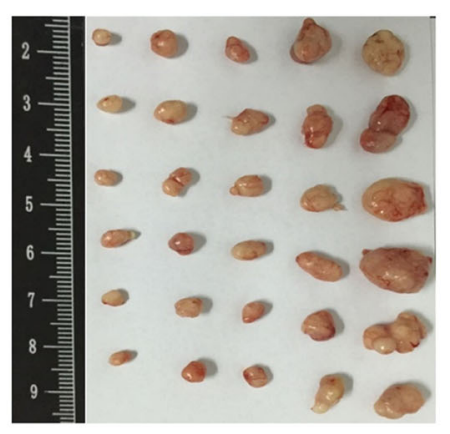

$\multimap-\backsim-\square-0-\neg$ b

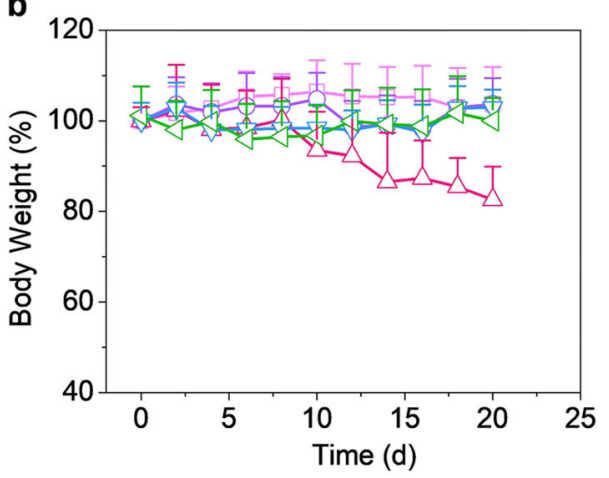

d

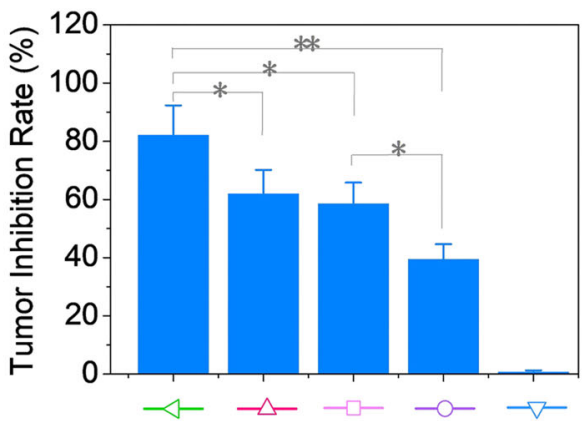

Fig. 4 Antitumor activity of CRGD-PS-Dox and PS-Dox in nude mice bearing subcutaneous A549 tumors. The drug was given on day 0, 4, 8, and 12 (Dox dose: 6 or $12 \mathrm{mg} / \mathrm{kg}$, in $200 \mu \mathrm{L}$ PBS). Lipo-Dox and PBS were used as controls. a Tumor volumes over 20 days. b Body weight changes over $20 \mathrm{~d}$. c Photographs of tumor blocks collected on day 20. d Tumor inhibition rate on day $20 .{ }^{*} p<0.05,{ }^{* *} p<0.01$ and ${ }^{* * *} p<0.001$ based on one-way ANOVA and Tukey multiple comparisons tests

therapeutic window compared with Lipo-Dox. A narrow therapeutic window is a key problem for chemotherapeutics and cancer nanomedicines, including Lipo-Dox. ${ }^{50,51}$ The in vivo studies in subcutaneous and orthotropic A549 lung tumor-bearing mice demonstrated that CRGD-PS-Dox can efficiently suppress tumor progression, significantly improve survival rates, and largely reduce side effects compared to Lipo-Dox and PS-Dox. Notably, when treated with $12 \mathrm{mg} / \mathrm{kg}$ cRGD-PS-Dox, the mice inoculated with orthotopic A549 lung tumors exhibited a median survival time of 57 days, which was significantly longer than that of LipoDox. The high potency of CRGD-PS-Dox can be attributed to its long circulation time, efficient and specific uptake by $a_{v} \beta_{3}$ integrin-overexpressing A549 cells, and triggered release of Dox in the cytoplasm, which contains $2-10 \mathrm{mM} \mathrm{GSH}$, leading to a greatly enhanced nucleic delivery of Dox. Dox is known to induce antitumor effects through intercalation into the DNA of cancer cells. ${ }^{52,53}$

In conclusion, we established that polymersomal doxorubicin cRGD-PS-Dox achieves an ultrahigh treatment efficacy in mice bearing subcutaneous or orthotropic human lung cancers with few adverse effects. Of note, CRGD-PS-Dox has several unique and desirable merits, such as high drug loading, a small size, superb stability, exceptional tolerability, high tumor accumulation and targetability, and modulated drug release. This polymersomal doxorubicin holds promise as a therapeutic agent for treating various $\alpha_{v} \beta_{3} / a_{v} \beta_{5}$ integrin-overexpressing tumors.

\section{MATERIALS AND METHODS}

In vivo pharmacokinetics of CRGD-PS-Dox

The mice were handled under protocols approved by Soochow University Laboratory Animal Center and the Animal Care and Use Committee of Soochow University. Tumor-free nude mice (18-20 $\mathrm{g}, n=3$ ) were i.v. injected with cRGD-PS-Dox (Dox dose: $10 \mathrm{mg} / \mathrm{kg}$ ) in $200 \mu \mathrm{L}$ PBS. Lipo-Dox, PS-Dox and PBS were used as controls. At set time points, approximately $50 \mu \mathrm{L}$ of blood was withdrawn from the eye sockets of mice, and Triton X-100 (1 wt\%, $0.1 \mathrm{~mL})$ was added immediately with brief sonication. To extract Dox. $\mathrm{HCl}, 0.5$ $\mathrm{mL}$ dimethyl formamide (DMF) solution containing $20 \mathrm{mM}$ dithiothreitol (DTT) was added, incubated for $6 \mathrm{~h}$ and stored at $-20^{\circ} \mathrm{C}$ overnight. After centrifugation (14.8k rpm, $15 \mathrm{~min}$ ), the supernatants were measured using fluorometry for Dox. $\mathrm{HCl}$ concentration. The blood circulation curves were drawn, and the half-lives were calculated as reported previously. ${ }^{21,41}$

Imaging of cRGD-PS-Dox in subcutaneous tumor xenografts Briefly, a subcutaneous A549 tumor model was established by injecting A549 cells $\left(1 \times 10^{7}\right.$ cells in $50 \mu \mathrm{L}$ PBS) into the right hind flank of nude mice (female, $18-20 \mathrm{~g}$ ). The tumor size reached approximately $150 \mathrm{~mm}^{3}$ after 15 days. For monitoring polymersomes in mice using fluorescence imaging, Cy7-labeled CRGD-PS and PS in $200 \mu \mathrm{L} \mathrm{PB}$, prepared by incorporating $5 \mathrm{~mol} \%$ Cy7labeled PEG-P(TMC-DTC) copolymer, were i.v. injected into randomly grouped tumor-bearing mice via tail vein. After $0,1,4$, $8,12,24$ or $48 \mathrm{~h}$, the mice were anesthetized and immediately imaged with an IVIS Lumina II with constant isoflurane (3\%) supply.

Biodistribution of cRGD-PS-Dox A subcutaneous A549 tumor xenograft model was established as described above. cRGD-PS-Dox, PS-Dox, or Lipo-Dox (Dox. $\mathrm{HCl}$ dose: $10 \mathrm{mg} / \mathrm{kg}$ ) or PBS was i.v. injected into randomly grouped mice bearing A549 subcutaneous tumor xenografts $(n=3)$. After $12 \mathrm{~h}$, tumors and major organs were excised, flushed, towel-dried and weighed before being subjected to fluorescence imaging. 
a
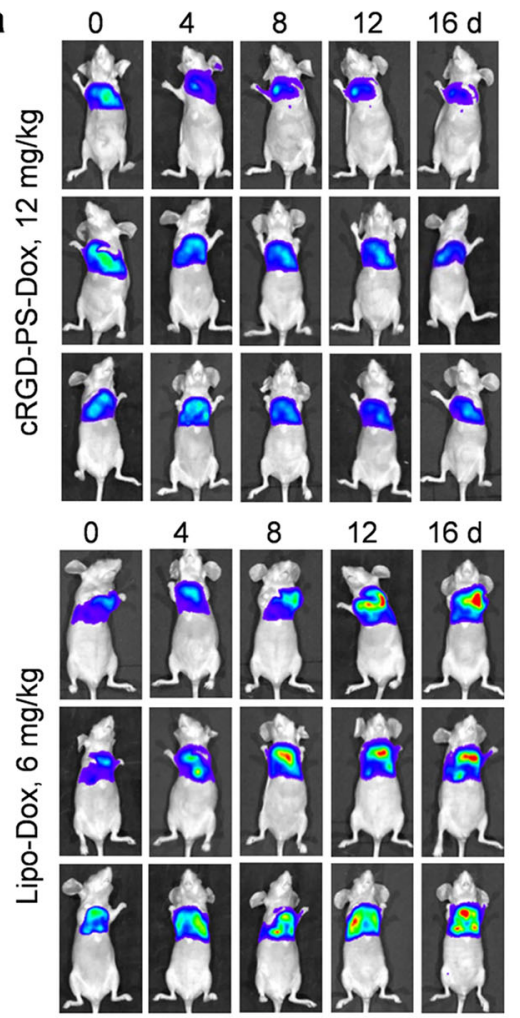

CRGD-PS-Dox, $6 \mathrm{mg} / \mathrm{kg}$ $\triangleleft$ - CRGD-PS-Dox, $12 \mathrm{mg} / \mathrm{kg}$
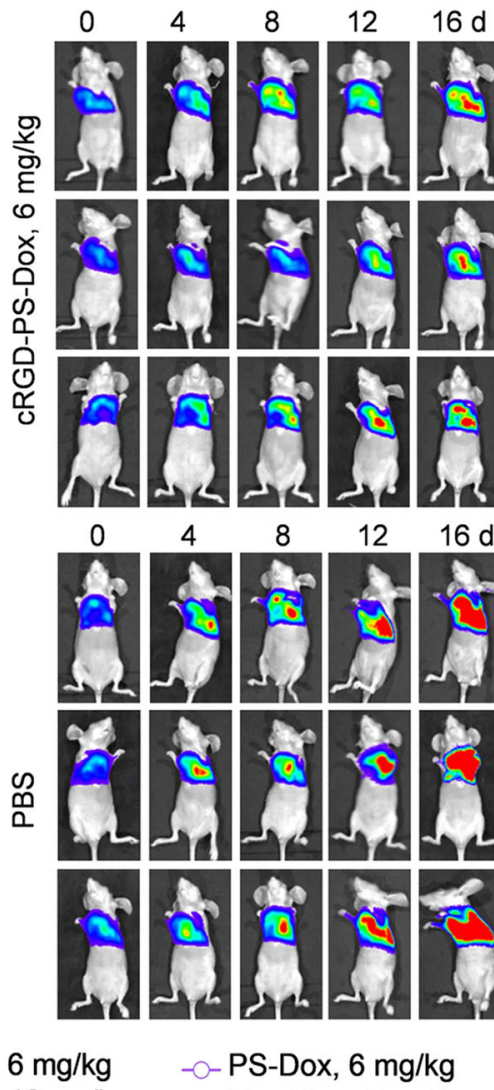

$\triangle$ Lipo-Dox, $6 \mathrm{mg} / \mathrm{kg}$

b

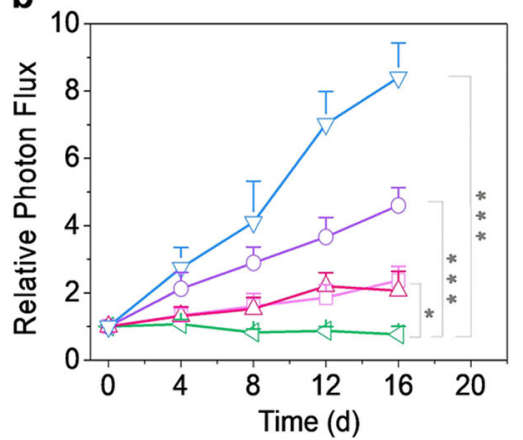

C

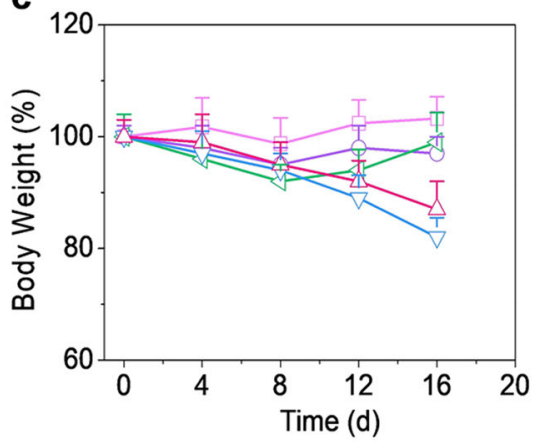

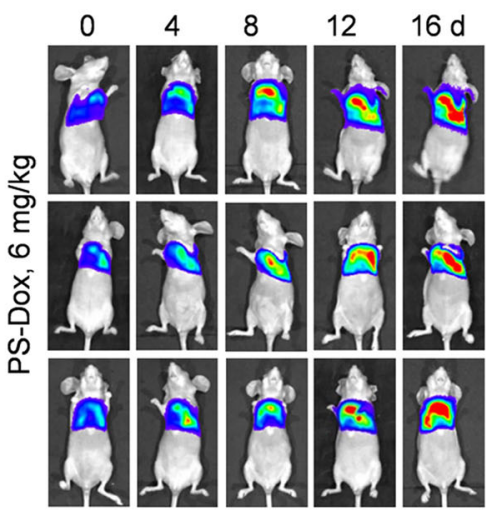

High

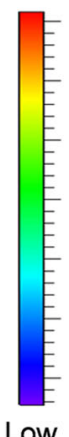

$\rightarrow$ PBS

d

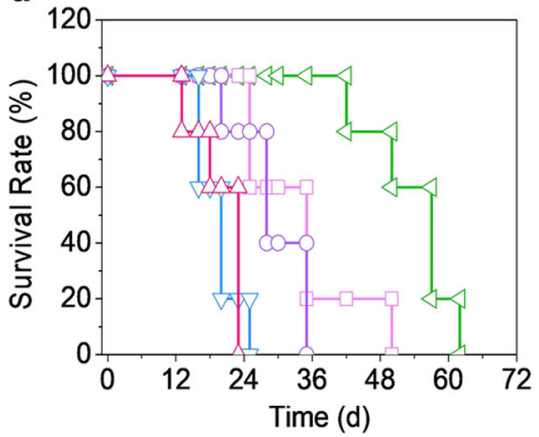

Fig. 5 In vivo antitumor activity of CRGD-PS-Dox, PS-Dox, and Lipo-Dox in nude mice bearing orthotopic A549 tumor xenografts at a Dox dose of 6 or $12 \mathrm{mg} / \mathrm{kg}$ in $200 \mu \mathrm{L}$ PBS administered on day $0,4,8$, and 12. a Bioluminescence images of mice over time. b Bioluminescence intensity of mice. One-way ANOVA and Tukey multiple comparisons tests, ${ }^{*} p<0.05$ and ${ }^{* * *} p<0.001$. c Body weight of mice over 16 days. d Survival curves of mice

The tumors and organs were subsequently homogenized (IKA T25, $18 \mathrm{k}$ rpm, $10 \mathrm{~min}$ ) in $600 \mu \mathrm{L}$ Triton $\mathrm{X}-100$ solution (1\%). An extraction solution ( $900 \mu \mathrm{L}$ DMF with $20 \mathrm{mM}$ DTT and $50 \mathrm{mM} \mathrm{HCl}$ ) was added to each tissue lysate, incubated for $6 \mathrm{~h}$ and stored at $-20^{\circ} \mathrm{C}$ overnight. After centrifugation, the supernatants were measured using fluorometry for Dox. $\mathrm{HCl}$ concentration, which was expressed as injected dose per gram of tissue (\%ID/g).

In vivo antitumor performance of cRGD-PS-Dox in subcutaneous lung tumor model

A mouse subcutaneous A549 tumor xenograft model was established as described above. Treatment started when the tumor volume reached $100 \mathrm{~mm}^{3}$ after 10 days, defined as day 0 . The mice were randomly assigned into five groups $(n=6)$ : cRGDPS-Dox (6 or $12 \mathrm{mg} / \mathrm{kg})$, PS-Dox (6 mg/kg), Lipo-Dox $(6 \mathrm{mg} / \mathrm{kg})$ and
PBS. The formulations and control were intravenously injected every 4 days (total 4 injections). The body weight and tumor size of the mice were measured every 2 days and normalized to their initial values on day 0 . On day 20 , the mice were sacrificed and tumors were excised and weighed. The tumor inhibition rates were calculated compared to the tumor weight of the PBS treated group.

In vivo antitumor efficacy of cRGD-PS-Dox in an orthotopic lung tumor model

A549-Luc cancer cells $\left(1 \times 10^{7}\right.$ in $100 \mu \mathrm{L}$ PBS) were injected into the lungs of female nude mice $(18-20 \mathrm{~g})$ through syringes. Tumor development was estimated using an IVIS Lumina system by visualizing cancer cells transfected by luciferase at 10-15 min after the injection of luciferin intraperitoneally $(150 \mathrm{mg}$ luciferin $/ \mathrm{kg}$ of 

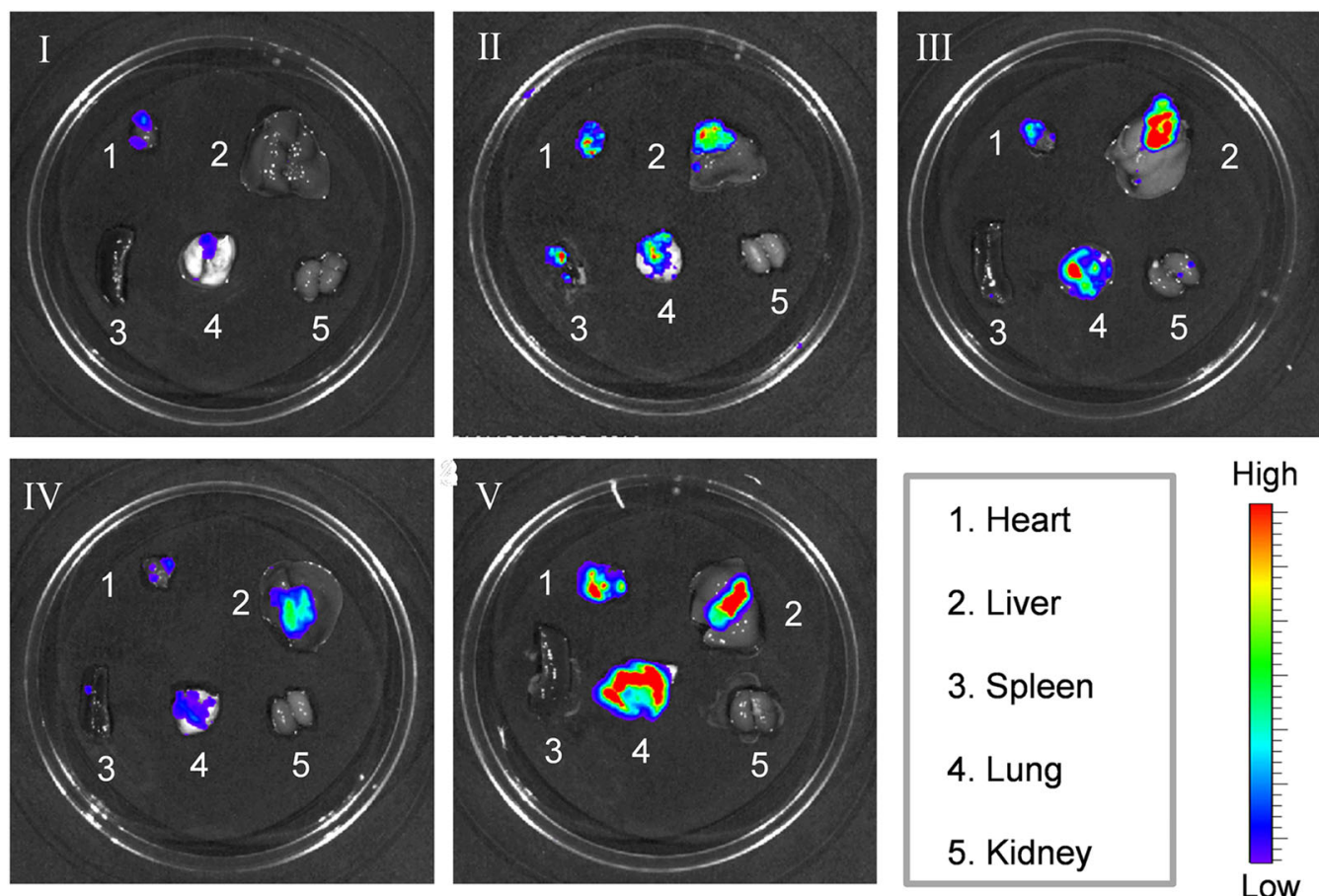

High

CRGD-PS-Dox@6 mg/kg, III. PS-Dox@6 mg/kg, IV. Lipo-Dox@6 mg/kg, V. PBS

body weight). Normally after 10 days, the tumor luminescent region of interest $(\mathrm{ROI})$ intensity of the mice reached approximately $1 \times 10^{4}-2 \times 10^{4}$. The grouping and dosing of the mice were the same as in the subcutaneous lung tumor model. The day treatment started was defined as day 0 . The mice were weighed every four days and normalized to their initial weights on day 0 . Every four days, the tumor bioluminescence was tracked using the IVIS Lumina system, and the relative photon flux was normalized to the initial intensity, $\mathrm{I} / \mathrm{I}_{0}\left(\mathrm{I}_{0}\right.$ is the bioluminescent intensity on day 0$)$. All images were set to the same conditions and color scale. On day 16, one mouse from each group was sacrificed after injection of luciferin. Major organs were excised, washed and imaged by an IVIS II instrument. The remaining mice were observed for survival curves $(n=5)$.

\section{Statistical analysis}

Differences between groups were assessed using the one-way ANOVA and Tukey multiple comparisons tests. Kaplan-Meier survival curves were analyzed by one-way ANOVA with a logrank test for comparisons. Data were analyzed using GraphPad Prism 7 and are expressed as the mean \pm SD. ${ }^{*} p<0.05$ was considered significant, and ${ }^{* *} p<0.01,{ }^{* *} p<0.001$ were considered highly significant.

\section{ACKNOWLEDGEMENTS}

This work was supported by research grants from the National Natural Science Foundation of China (NSFC 51633005, 51773146, 51561135010, 51473111).

\section{AUTHOR CONTRIBUTIONS}

Y.Z., F.M., and Z.Z. conceived and designed the experiments. Y.Z., J.W., and Y.X. performed the experiments. Y.Z., F.M., J.Y., and Z.Z. co-wrote the paper. All of the authors discussed the results and commented on the manuscript.

\section{ADDITIONAL INFORMATION}

The online version of this article (https://doi.org/10.1038/s41392-018-0032-7) contains supplementary material, which is available to authorized users.

Competing interests: The authors declare no competing interests.

\section{REFERENCES}

1. Lortet-Tieulent, J. et al. International trends in lung cancer incidence by histological subtype: adenocarcinoma stabilizing in men but still increasing in women. Lung Cancer 84, 13-22 (2014).

2. Anagnostou, V. et al. Evolution of neoantigen landscape during immune checkpoint blockade in non-small cell lung cancer. Cancer Discov. 7, 264-276 (2017).

3. Brahmer, J. et al. Nivolumab versus docetaxel in advanced squamous-cell non-small-cell lung cancer. N. Engl. J. Med. 373, 123-135 (2015).

4. Shaw, A. T. et al. Crizotinib versus Chemotherapy in Advanced ALK-Positive Lung Cancer. N. Engl. J. Med. 368, 2385-2394 (2013).

5. Wu, Y.-L. et al. Afatinib versus cisplatin plus gemcitabine for first-line treatment of Asian patients with advanced non-small-cell lung cancer harbouring EGFR mutations (LUX-Lung 6): an open-label, randomised phase 3 trial. Lancet Oncol. 15, 213-222 (2014).

6. Nawaz, K. \& Webster, R. M. The non-small-cell lung cancer drug market. Nat. Rev. Drug. Discov. 15, 229-230 (2016).

7. Jiang, L. et al. Overcoming drug-resistant lung cancer by paclitaxel loaded dualfunctional liposomes with mitochondria targeting and $\mathrm{pH}$-response. Biomaterials 52, 126-139 (2015).

8. Patel, K. et al. Tumor stromal disrupting agent enhances the anticancer efficacy of docetaxel loaded PEGylated liposomes in lung cancer. Nanomedicine 11, 1377-1392 (2016).

9. Yang, Y. et al. Tumor-responsive small molecule self-assembled nanosystem for simultaneous fluorescence imaging and chemotherapy of lung cancer. Adv. Funct. Mater. 26, 8735-8745 (2016).

10. Zhang, Z. et al. Construction of a supramolecular drug-drug delivery system for non-small-cell lung cancer therapy. ACS Appl. Mater. Interfaces 9, 29505-29514 (2017).

11. Zou, Y. et al. Virus-mimicking chimaeric polymersomes boost targeted cancer siRNA therapy in vivo. Adv. Mater. 29, 1703285 (2017).

12. Yang, W. et al. Efficient and targeted suppression of human lung tumor xenografts in mice with methotrexate sodium encapsulated in all-function-in-one chimeric polymersomes. Adv. Mater. 28, 8234-8239 (2016). 
13. Lv, S. et al. Co-delivery of doxorubicin and paclitaxel by PEG-polypeptide nanovehicle for the treatment of non-small cell lung cancer. Biomaterials 35, 6118-6129 (2014)

14. Senapati, S., Mahanta, A. K., Kumar, S. \& Maiti, P. Controlled drug delivery vehicles for cancer treatment and their performance. Signal Transduct. Target Ther. 3, 7 (2018).

15. Han, W. et al. A nanomedicine approach enables co-delivery of cyclosporin A and gefitinib to potentiate the therapeutic efficacy in drug-resistant lung cancer. Signal Transduct. Target Ther. 3, 16 (2018).

16. Wang, $\mathrm{H}$. et al. New generation nanomedicines constructed from self-assembling small molecule prodrugs alleviate cancer drug toxicity. Cancer Res. 77, 6963-6973 (2017).

17. Wang, $\mathrm{H}$. et al. Polylactide-tethered prodrugs in polymeric nanoparticles as reliable nanomedicines for the efficient eradication of patient-derived hepatocellular carcinoma. Theranostics 8, 3949-3963 (2018).

18. Wang, $H$. et al. Cancer nanomedicines stabilized by $\pi-\pi$ stacking between heterodimeric prodrugs enable exceptionally high drug loading capacity and safer delivery of drug combinations. Theranostics 7, 3638-3652 (2017).

19. Ahn, H. K. et al. A phase II trial of Cremorphor EL-free paclitaxel (Genexol-PM) and gemcitabine in patients with advanced non-small cell lung cancer. Cancer Chemoth. Pharm. 74, 277-282 (2014).

20. Pearce, T. R., Shroff, K. \& Kokkoli, E. Peptide targeted lipid nanoparticles for anticancer drug delivery. Adv. Mater. 24, 3803-3822 (2012).

21. Zou, Y., Meng, F., Deng, C. \& Zhong, Z. Robust, tumor-homing and redox-sensitive polymersomal doxorubicin: A superior alternative to Doxil and Caelyx? J. Control Release 239, 149-158 (2016).

22. Yang, W. et al. Bioresponsive chimaeric nanopolymersomes enable targeted and efficacious protein therapy for human lung cancers in vivo. Chem. Mater. 29, 8757-8765 (2017).

23. Yang, W. et al. Lung cancer specific and reduction-responsive chimaeric polymersomes for highly efficient loading of pemetrexed and targeted suppression of lung tumor in vivo. Acta Biomater. 70, 177-185 (2018).

24. Talelli, M. et al. Core-crosslinked polymeric micelles: principles, preparation, biomedical applications and clinical translation. Nano Today 10, 93-117 (2015).

25. Jiang, Y., Yang, W., Zhang, J., Meng, F., Zhong, Z. Protein toxin chaperoned by LRP-1-targeted virus-mimicking vesicles induces high-efficiency glioblastoma therapy in vivo. Adv. Mater., https://doi.org/10.1002/adma.201800316 (2018).

26. Zou, Y. et al. Self-crosslinkable and intracellularly decrosslinkable biodegradable micellar nanoparticles: a robust, simple and multifunctional nanoplatform for high-efficiency targeted cancer chemotherapy. J. Control Release 244, 326-335 (2016).

27. Dong, H. et al. Disulfide-bridged cleavable PEGylation in polymeric nanomedicine for controlled therapeutic delivery. Nanomedicine 10, 1941-1958 (2015).

28. Zhu, Y. et al. Highly efficacious and specific anti-glioma chemotherapy by tandem nanomicelles co-functionalized with brain tumor-targeting and cell-penetrating peptides. J. Control Release 278, 1-8 (2018).

29. Yang, W. et al. Selective cell penetrating peptide-functionalized polymersomes mediate efficient and targeted delivery of methotrexate disodium to human lung cancer in vivo. Adv. Healthc. Mater. 7, 1701135 (2018).

30. Barenholz, Y. C. Doxil ${ }^{-}$-the first FDA-approved nano-drug: lessons learned. J. Control Release 160, 117-134 (2012).

31. Balasubramanian, V., Herranz-Blanco, B., Almeida, P. V., Hirvonen, J. \& Santos, H. A. Multifaceted polymersome platforms: Spanning from self-assembly to drug delivery and protocells. Prog. Polym. Sci. 60, 51-85 (2016).

32. Wibroe, P. P., Ahmadvand, D., Oghabian, M. A., Yaghmur, A. \& Moghimi, S. M. An integrated assessment of morphology, size, and complement activation of the PEGylated liposomal doxorubicin products Doxil $^{\circledR}$, Caelyx $^{\oplus}$, DOXOrubicin, and SinaDoxosome. J. Control Release 221, 1-8 (2016).

33. Gabizon, A. A., Patil, Y. \& La-Beck, N. M. New insights and evolving role of pegylated liposomal doxorubicin in cancer therapy. Drug Resist. Update 29, 90-106 (2016).

34. Russell, L. M., Hultz, M. \& Searson, P. C. Leakage kinetics of the liposomal chemotherapeutic agent Doxil: The role of dissolution, protonation, and passive transport, and implications for mechanism of action. J. Control Release 269, 171-176 (2017).
35. Deng, Y., Ling, J. \& Li, M. H. Physical stimuli-responsive liposomes and polymersomes as drug delivery vehicles based on phase transitions in the membrane. Nanoscale 10, 6781-6800 (2018).

36. Zhao, L. et al. Polyglycerol-coated nanodiamond as a macrophage-evading platform for selective drug delivery in cancer cells. Biomaterials 35, 5393-5406 (2014).

37. Fan, R. et al. Self-assembled bifunctional peptide as effective drug delivery vector with powerful antitumor activity. Adv. Sci. 4, 1600285-n/a (2017).

38. Khatri, N., Baradia, D., Vhora, I., Rathi, M. \& Misra, A. CRGD grafted liposomes containing inorganic nano-precipitate complexed siRNA for intracellular delivery in cancer cells. J. Control Release 182, 45-57 (2014).

39. Qiu, M. et al. Lipopepsomes: A novel and robust family of nano-vesicles capable of highly efficient encapsulation and tumor-targeted delivery of doxorubicin hydrochloride in vivo. J. Control Release 272, 107-113 (2018).

40. Wang, Y. et al. A nanoparticle-based strategy for the imaging of a broad range of tumours by nonlinear amplification of microenvironment signals. Nat. Mater. 13, 204-212 (2014).

41. Fang, Y. et al. EGFR-targeted multifunctional polymersomal doxorubicin induces selective and potent suppression of orthotopic human liver cancer in vivo. Acta Biomater. 64, 323-333 (2017).

42. Hassan, S. et al. Evolution and clinical translation of drug delivery nanomaterials. Nano Today 15, 91-106 (2017).

43. Shi, Y. et al. Complete regression of xenograft tumors upon targeted delivery of paclitaxel via П-П stacking stabilized polymeric micelles. ACS Nano 9, 3740-3752 (2015).

44. Miura, Y. et al. Cyclic RGD-linked polymeric micelles for targeted delivery of platinum anticancer drugs to glioblastoma through the blood-brain tumor barrier. ACS Nano 7, 8583-8592 (2013).

45. Kunjachan, S. et al. Passive versus active tumor targeting using RGD-and NGRmodified polymeric nanomedicines. Nano Lett. 14, 972-981 (2014).

46. Chen, X. et al. Integrin aß3-targeted imaging of lung cancer. Neoplasia 7, 271-279 (2005).

47. Chen, H., Niu, G., Wu, H. \& Chen, X. Clinical application of radiolabeled RGD peptides for PET imaging of integrin $a(v) \beta(3)$. Theranostics 6, 78-92 (2016).

48. Sun, X. et al. Peptide-based imaging agents for cancer detection. Adv. Drug Deliv. Rev. 110-111, 38-51 (2017).

49. Ma, X., Jia, J., Cao, R., Wang, X. \& Fei, H. Histidine-iridium(III) coordination-based peptide luminogenic cyclization and cyclo-RGD peptides for cancer-cell targeting. J. Am. Chem. Soc. 136, 17734-17737 (2014).

50. Hare, J. I. et al. Challenges and strategies in anti-cancer nanomedicine development: An industry perspective. Adv. Drug Deliv. Rev. 108, 25-38 (2017).

51. Tibbitt, M. W., Dahlman, J. E. \& Langer, R. Emerging frontiers in drug delivery. J. Am. Chem. Soc. 138, 704-717 (2016).

52. Marino Gammazza, A. et al. Doxorubicin anti-tumor mechanisms include Hsp60 post-translational modifications leading to the Hsp60/p53 complex dissociation and instauration of replicative senescence. Cancer Lett. 385, 75-86 (2017).

53. Carrassa, L. \& Damia, G. DNA damage response inhibitors: mechanisms and potential applications in cancer therapy. Cancer Treat. Rev. 60, 139-151 (2017).

Open Access This article is licensed under a Creative Commons Attribution 4.0 International License, which permits use, sharing, adaptation, distribution and reproduction in any medium or format, as long as you give appropriate credit to the original author(s) and the source, provide a link to the Creative Commons license, and indicate if changes were made. The images or other third party material in this article are included in the article's Creative Commons license, unless indicated otherwise in a credit line to the material. If material is not included in the article's Creative Commons license and your intended use is not permitted by statutory regulation or exceeds the permitted use, you will need to obtain permission directly from the copyright holder. To view a copy of this license, visit http://creativecommons. org/licenses/by/4.0/.

(c) The Author(s) 2018 